\title{
Materials Pursuit Gathers Steam at the 1991 MRS Fall Meeting
}

Both heated debate and quiet scientific progress filled the 1991 Fall Meeting held December 2-6, 1991 at Boston's Copley Place, all with the intent of resolving mysteries and piecing together puzzles often hidden in contradictions.

Leading the list of hot topics-both in interest and debate-was light emission from porous silicon, addressed in a late-news session which exploded into an evening session, two poster sessions, a full day of presentations, and finally a panel discussion. Potential device applications wait eagerly in the wings while scientists unabashedly expressed their differing views as to why it works.

In contrast, the late-news session on fullerenes showed how the versatility of fullerenes continues to expand without limit, with research moving in every direction, not toward a particular application, but defining a whole new field of scientific pursuit.

Other topics drawing interest and lively discussion included cluster beams, precipitates in GaAs grown at low temperatures and their role in producing interesting electrical properties, and diamond growth on single-crystal substrates using ion beams.

In-situ observation of surfaces and interfaces gave researchers front-row seats for seeing growth and etching of materials as it happens, leaping from the age of still photography to the age of movies. Ferroelectrics are now being integrated with semiconductor devices, etching a path to form micromotors and other integrated devices. Meanwhile, SQUID technology for high-temperature superconductors push these superconductors closer to maturity.

Hidden beneath the spirited technical hum, however, was a low murmur concerned about the difficult economic times and the restructuring happening in many companies and national laboratories. Yet, this meeting was the largest MRS meeting ever, with over 4,000 attendees and 2,700 abstracts accepted, which were distributed among 26 symposia.

- Plenary Address. D. Allan Bromley, Assistant to the President for Science and
Technology and Director of the Office of Science and Technology Policy in the Executive Office of the President, gave the plenary address. He described the federal government's current role in policy making, goal setting, funding, and collaborative research and development efforts in materials science. He looked at future needs in materials science, setting them in the context of broader U.S. science and technology goals. His plenary talk is published in this issue's Material Matters column.

- Von Hippel Address. In his Von Hippel Award presentation, Theodore Geballe of Stanford University asked "Why don't we have a room-temperature superconductor (yet)?" He vividly traced the history of superconductors from A15 and cubic pervoskites to cuprate structures, fullerenes, and organic charge transfer salts. The search for a room-temperature superconductor, Geballe says, is somewhat like the extraterrestrial search; there are a lot of galaxies out there, just like there are many metastable compounds, and we certainly haven't exhausted them all. Geballe's presentation will appear in a future issue of the MRS Bulletin. See Editor's Choice in this issue for additional commentary on high-temperature superconductors.

- Symposium X. This lunchtime session presented animated reviews for the nonspecialist on polymers, nanofabrication, ion/solid interaction, biomaterials, shapememory alloys, diamond films, plus presentations by the two MRS medalists, Shigeyuki Sōmiya (Nishi Tokyo University) on hydrothermal synthesis of ceramics and Bernard S. Meyerson (IBM) on Si-Ge high-speed heterojunction transistors.

- Technical Sessions. For a glimpse of what went on in specific technical sessions, read the summaries published here. More detailed information is available in the published proceedings (listed elsewhere in this issue).

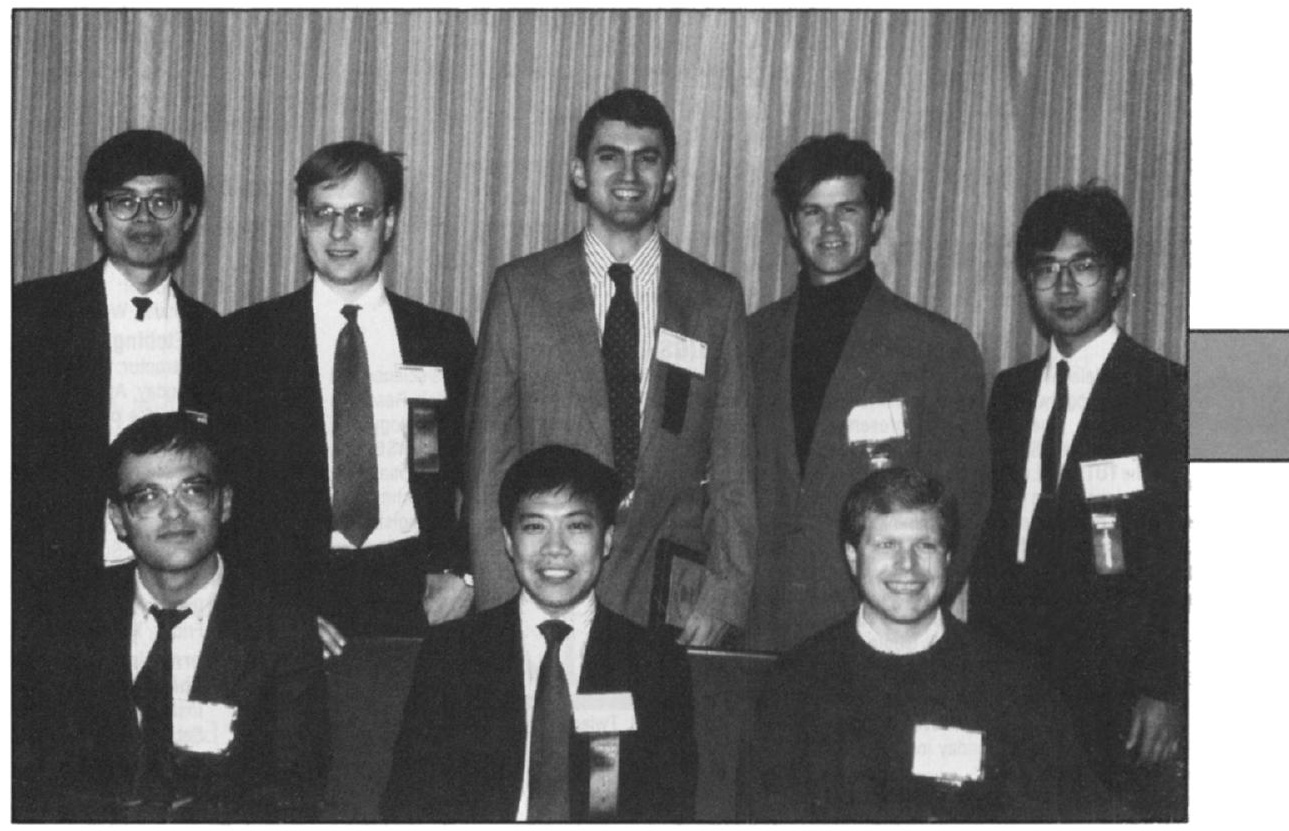

Graduate Student Award Winners at the 1991 MRS Fall Meeting. Front row, left to right: Ken Khachaturyan, University of California, Berkeley; Jiang Yue, Ohio State University; Brian L. Olmsted, University of Rochester. Second row, left to right: Jung-Chun Andrew Huang, University of Illinois at Urbana-Champaign; Samuel P. Gido, Massachusetts Institute of Technology; George Stejic, University of Wisconsin-Madison; Christopher P. Burmester, University of California; Yasukazu Murakami, University of Tsukuba, Japan. 

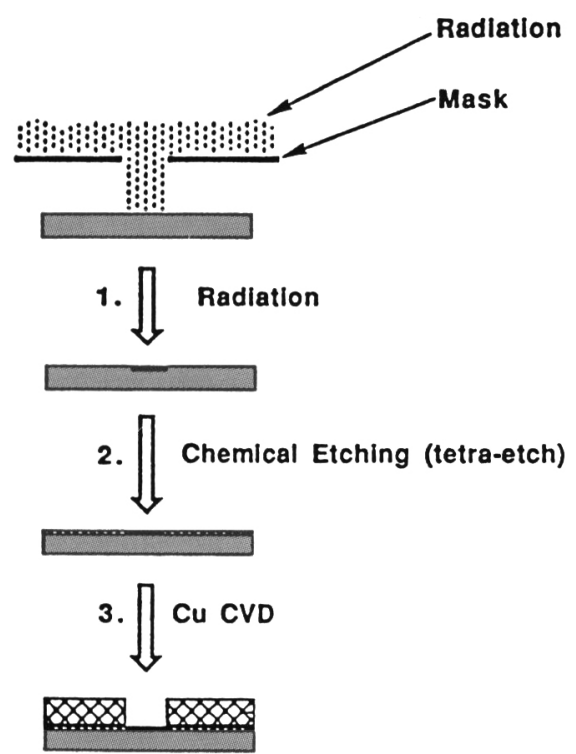

Copper can be deposited on Teflon in a three-step process. First, the Teflon surface where no copper is desired is irradiated through a mask. Second, the material is chemically etched, causing only un-irradiated portions to etch. Last, copper is deposited by $C V D$, selectively attaching only on the etched surfaces. (Presentation B3.4, Robert R. Rye, Sandia National Laboratories.)

(Photons and Low Energy Particles in Surface Processing) and $\mathrm{H}$ (High Temperature Superconductors).

Two of the most notable topics covered in this symposium were the formation of diamond-like films by ion implantation and the use of ion beams to study flux pinning in superconductors.

Recently, there has been significant interest in the possibility of producing diamond on single-crystal copper by ion implantation followed by laser annealing. Initial reports of the formation of diamond films have been tempered somewhat by unsuccessful attempts to repeat these experiments. These were reported in the symposium and have fueled the controversy over the possibility of synthesizing diamond on single crystal substrates by implantation.

Enhanced pinning in superconductors by ion irradiation was highlighted by results which show that in contrast to initial investigation with light ions or neutrons, pinning is most effectively produced by very energetic heavy ions. Pinning of the magnetic flux lines helps increase the critical current of high temperature superconductors by reducing the resistance caused by the motion of the flux lines. High energy, heavy ions (such as $580 \mathrm{MeV}$ Sn) produce a different shape pinning defect than lighter ions, and pin more efficiently. As a consequence, at temperatures near that of liquid nitrogen, the potentially useful magnetic field range in which the critical current exceeds has been enlarged by several Tesla.

\section{Lasers, Low Energy Particles Lead to Improved Surface Processing (See MRS Proceedings Vol. 236)}

The four-day Symposium on Photons and Low Energy Particles in Surface Processing (Symposium B) featured laser etching, ablation and film deposition, electron beam induced decomposition and growth, plasma etching, and ion-induced effects.

Teflon processing was a subject of two interesting papers. In a presentation by $\mathrm{M}$. Okoshi of Tokai University in Japan, the formation of a $\mathrm{SiC}$ surface layer by excimer laser irradiation of Teflon in the presence of gaseous $\mathrm{B}\left(\mathrm{CH}_{3}\right)_{3}$ and $\mathrm{SiH}_{4}$ was described. In a second report, $\mathrm{R}$. Rye of Sandia $\mathrm{Na}$ tional Laboratories, Albuquerque, NM, gave details on a novel means of patterndepositing copper onto Teflon, followed by a sodium-containing liquid etch, permitting copper deposition in the nonirradiated regions.

The use of low-energy ions for process control and minimizing atomic-displacement damage was featured in several presentations. Modified versions of the ion-solid interaction computer code TRIM (TRansport of Ions in Matter) to model the effects of low-energy ion sputtering and damage were used to explain experimental data. H. Winters of IBM Almaden Research, San Jose, CA, described a novel calorimeter technique which determines the amount of ion-beam energy reflected from a surface as a function of incident ion energy. At incident argon ion energies of less than $500 \mathrm{eV}$, gold or platinum surfaces become mirror like, reflecting nearly all of the incident energy. Such high reflectivity poses problems for low-energy ion processing techniques.

Several aspects of laser ablation were discussed in various presentations. An incoherent excimer lamp was described by $\mathrm{H}$. Esrom of Asea Brown Boveri AG, Heidelberg, Germany. The ability of this lamp to etch PMMA at $308 \mathrm{~nm}$ and Teflon at 222 $\mathrm{nm}$ elicited active discussion among participants. R. Singh of the University of Florida, Gainesville, discussed film thickness uniformity considerations in films deposited by laser ablation, showing how laser spot sizes of $<10^{-2} \mathrm{~mm}$ provide greater uniformity than large spots. P.T. Murray of the University of Dayton, Ohio, evoked inter- est over his presentation on the ionic and neutral precursors to diamond-like carbon film growth originating from excimer laser ablation of graphite.

Laser ablation was also the theme in a poster presentation by $\mathrm{M}$. Vollmer of the Universität Kassel, Germany. Vollmer's discussion of a novel "nonlocal" optical effect to account for nonthermal ablation of metallic clusters won for him the Best Poster Presentation Award.

\section{Thin Film Stresses Studied in New Ways} (See MRS Proceedings Vol. 239)

The symposium on Thin Films: Stresses and Mechanical Properties (Symposium D) dealt with both fundamental and applied problems. The symposium featured 9 invited speakers, 73 contributed oral presentations and over 50 poster presentations. Speakers and poster presenters came from 14 different countries. The symposium was the third in a series that has been stimulated by a growing awareness of the importance of stresses and mechanical properties in thin film technologies and by the recent introduction of new experimental techniques for studying stresses and mechanical properties of thin films.

Motivation for understanding stresses and mechanical properties of thin films was provided by Stephen Senturia of the Massachusetts Institute of Technology who reviewed the physical processes that lead to residual stresses in thin films and discussed progress that has been made in predicting process-dependent properties of thin films. Other contributed papers emphasized the role of microstructural processes in determining intrinsic stresses in thin films. The fundamental origins of stresses in thin films deposited under ultrahigh vacuum conditions were discussed by R. Abermann of the University of Innsbruck who showed that very small amounts of oxygen can have powerful effects on the development of stresses in metal films.

A number of invited speakers discussed different kinds of deformation processes that can occur in thin films. Frans Spaepen of Harvard University discussed viscous deformation in amorphous thin films and showed that structural relaxation of amorphous films can be studied by monitoring its effects on viscosity. Anelastic deformation and damping of thin metal films was described by H.G. Bohn of Jülich. He showed that internal friction techniques can be used to detect grain boundary sliding and diffusion in thin metal films. Such techniques can be used to characterize metallizations and to predict the probable resistance of a particular metallization to failure by electromigration. 


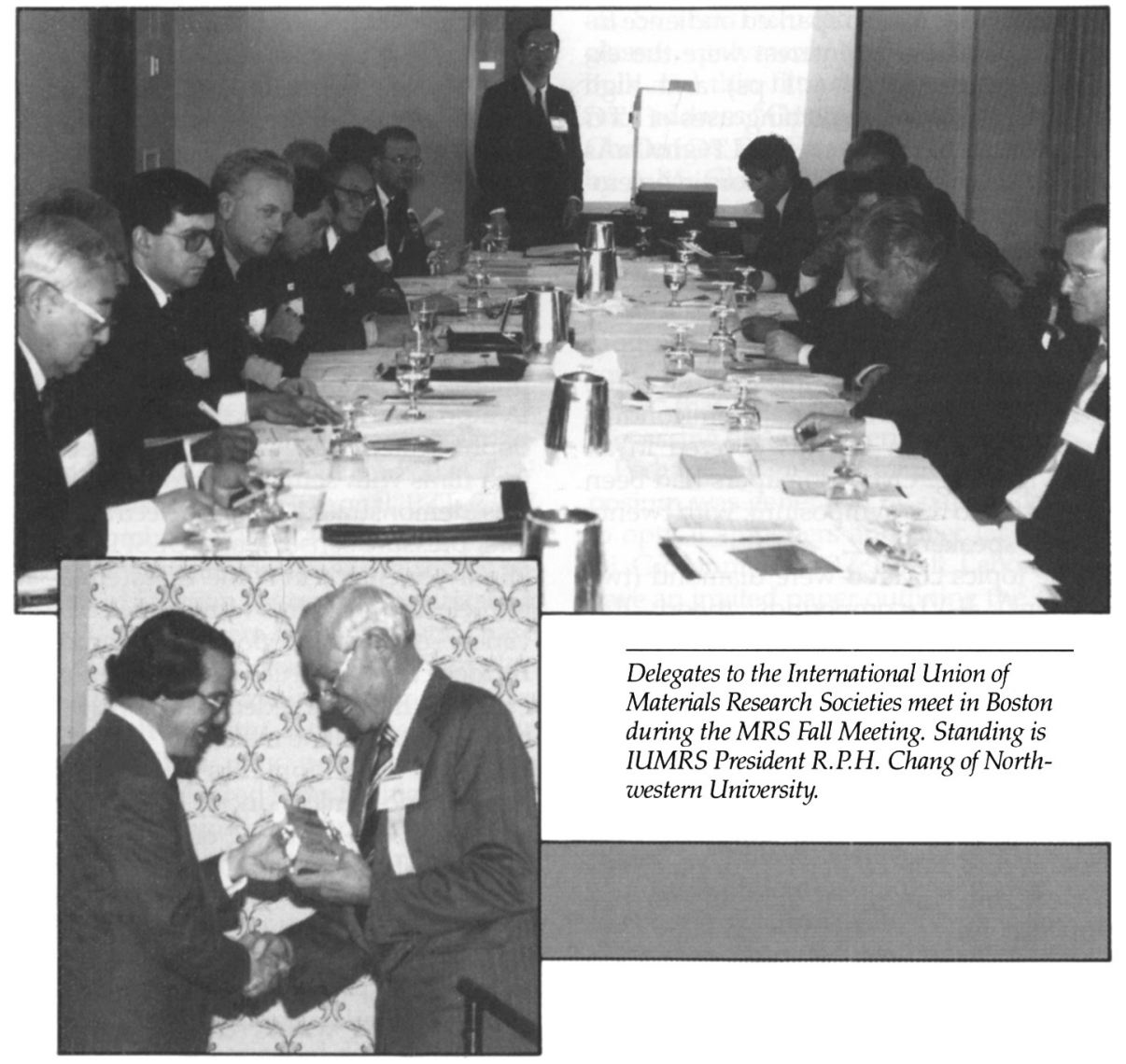

Theodore H. Geballe (right), emeritus professor in Stanford University's Departments of Applied Physics and Materials Science and Engineering, accepts the Von Hippel Award from 1991 MRS President Jim Roberto. Geballe's address, "Why Don't We Have a Room-Temperature Superconductor (Yet)?" will be published in an upcoming issue of the MRS Bulletin.

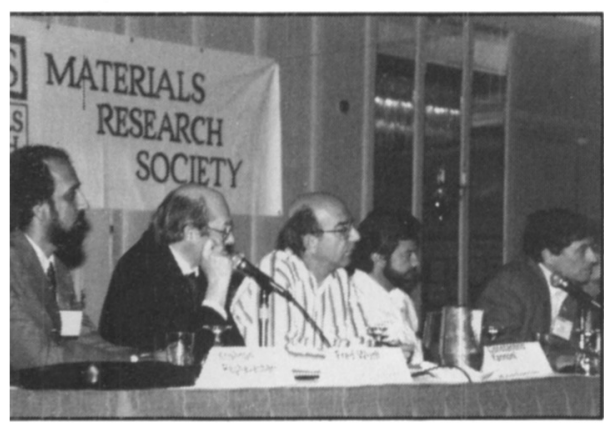

Panel discussion of fullerenes wraps up the latest news on this topic.

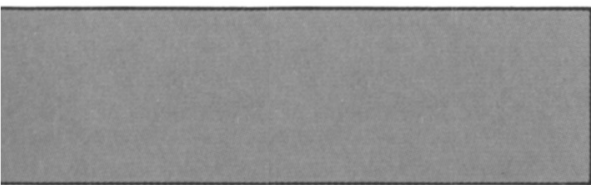

Progress in understanding the mechanics of indentation and the indentation properties of thin films was reviewed in a series of papers. G.M. Pharr of Rice University pointed out that the indentation of silicon, so commonly reported in the literature, should be regarded as anomalous, both because a phase transformation can be induced under the indenter and because cracking can occur during the indentation process. Other papers emphasized the growing importance of stiffness measurements in the determination of contact areas during indentation. The measurement and interpretation of rate-sensitive deformation behavior of thin films was also described in several papers.

Robert Hull of AT\&T Bell Laboratories reported on the advances made in understanding strain relaxation by misfit dislocation formation in heteroepitaxial thin films. He showed that the mechanisms of misfit dislocation formation in SiGe films can be revealed more completely by studying films grown on (110) and (111) substrates, rather than the usual (001) substrates. The slip systems activated in these differently oriented films allows a study of the role of both partial and perfect dislocations in strain relaxation. Two groups reported on in-situ measurements of strain relaxation in Si-Ge films at high tempera- tures using wafer curvature measurements. These kinds of measurements permit a study of the evolution of the density of moving dislocations during strain relaxation. A particularly dramatic report was given by $R$. Stalder of the ETH in Zuirich who showed that scanning tunneling microscopy could be used to image misfit dislocations located at $\mathrm{CoSi}_{2} / \mathrm{Si}$ epitaxial interfaces.

The structure and properties of metal multilayers were described in papers by B.M. Clemens of Stanford and I.K. Schuller of the University of California, San Diego. Clemens reported on recent advances in the use of $x$-ray diffraction techniques to determine the stress states in multilayer structures. He showed that the stresses in $\mathrm{Mo} / \mathrm{Ni}$ multilayers arise mainly from coherency effects and not from interfacial or capillarity effects. Schuller reported on the effect of structure on the mechanical properties of multilayers. He noted that anomalous elastic properties can be exhibited by these materials even though large enhancements in the elastic properties are not found. Nevertheless, the yield strengths of these materials can still be considered anomalously large, especially when compared with ordinary bulk materials.

Failure processes in thin films were also discussed at the symposium. A number of papers focused on the cracking of thin films and the delamination of thin films from their substrates. One particular failure mechanism, electromigration, received special attention. J.R. Lloyd of Digital Equipment Corporation reviewed the present state of understanding of electromigration failure mechanisms, emphasizing the important connection between the development of stresses in metallization lines and electromigration failure. A number of contributed papers focused on different aspects of the development of mechanical stresses in metal lines at points of flux divergence and the resulting nucleation and growth of voids.

\section{Fully Scalable Process Developed for Manufacturing Very High Speed AlInAs/InGaAs HBT}

(See MRS Symposium Proceedings Vol. 240)

Searching for new organometallic precursors for gas-source growth and using manufacturable dry etch processes for device and circuit fabrication were main themes in Symposium E on Advanced IIIV Compound Semiconductor Growth, Processing and Devices. In this large symposium, over 158 papers were presented in oral or poster form.

An invited presentation by D.R. 
Abernathy (AT\&T Bell Labs) highlighted the development of new approaches to metal organic molecular beam epitaxy using novel growth precursors and plasmaenhanced dissociation of these chemicals. T.R. Fullowan (AT\&T Bell Labs) presented details of a highly self-aligned, electron cyclotron resonance (ECR) dry etch process for fabricating very high speed AllnAs/ InGaAs heterojunction bipolar transistors. A scanning electron micrograph of a completed device is shown. This is the first fully scalable, manufacturable process for these devices, and represents an important breakthrough. Other sessions on implantation, ohmic contacts, deposition, and characterization were well attended with lively debate.

\section{III-V Compounds Grown at Low Temperatures Display Unusual Properties}

(See MRS Symposium Proceedings Vol. 241)

Low-temperature grown (LTG) GaAs, InP, and InGaAs captured the attention of the 150-200 people attending Symposium F. These materials are obtained by epitaxial growth in an MBE system, at an unusually low substrate temperature. The materials contain an exceptionally large nonstoichiometric excess of the group $\mathrm{V}$ element. Stimulating questions and discussions followed nearly every speaker. Particularly interesting was the controversial issue of the physical models used to explain the electrical properties of LTG GaAs. Advocates of the precipitate depletion-layer model argued strongly that their model could explain all of the observed LTG GaAs properties. Advocates of the deep-level model did concede that precipitates may be required to explain some of the device properties observed when LTG GaAs was used but that the deep levels were required to explain some of its electrical and optical properties. Another interesting topic was the segregation properties of As precipitates to the GaAs in AlAs/GaAs multilayer structures. This unexpected result stimulated much speculation on new device structures.

There were several reports of the incorporation of enormous numbers $\left(10^{20} \mathrm{~cm}^{-3}\right)$ of $P$ precipitates in LTG $\mathrm{InP}$ and one report on the surprising result of obtaining semiinsulating $\left(10^{5} \Omega \mathrm{cm}\right)$ LTG InP when an acceptor was included in the growth. The general consensus in the ensuing audience discussion was that assuming the excess $P$ created antisite defects in the $\ln \mathrm{P}$, the acceptor concentration would have to be controlled within a factor of two of the defect concentration for the InP to become insulating. The truth remains to be seem.

Device applications of the LTG GaAs and LTG InGaAs also sparked audience interest. Of particular interest were the extremely high speed ( $<1 \mathrm{ps)}$ and high voltage $(\sim 1,000 \mathrm{~V})$ switching uses of LTG GaAs and the effectiveness of LTG InGaAs as a diffusion barrier to acceptors in heterojunction bipolar transistors.

\section{Wide Band Gap Semiconductors Emit Blue Light \\ (See MRS Proceedings Vol. 242)}

This fall was the first time that the entire spectrum of wide band gap semiconductors (Symposium G) was covered in an MRS meeting. Over 130 papers had been submitted to this symposium, with twenty invited speakers.

The topics covered were diamond (two sessions), II-VI compounds, theory, II-V nitrides, boron compounds, silicon carbide, amorphous and microcrystalline semiconductors, and chalcopyrites, oxides, and halides.

The symposium addressed issues related to crystal growth (bulk and thin films), structure and microstructure, formation and role of defects, doping, optoelectronic properties and device applications.

A group from RTI (R.A. Rudder et al.) succeeded in making diamond films by plasma deposition at temperatures as low as $300^{\circ} \mathrm{C}$ using alcohol and water vapors as described in a post-deadline paper. A presentation by the Purdue-Brown consortium (R.L. Gunshore et al.) described bright blue injection luminescence from multiple quantum wells in a $\mathrm{ZnSe}$ double heterojunction at liquid nitrogen temperatures. A group at Nagoya University (I.

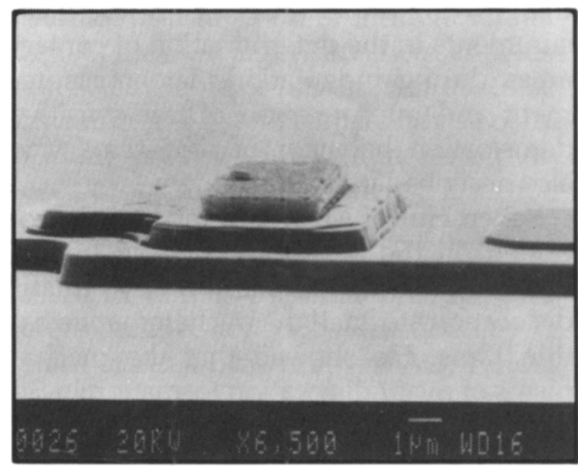

Scanning electron micrograph of a highly self-aligned, electron cyclotron resonance $(E C R)$ dry etch process for fabricating very high-speed AlInAs/InGaAs heterojunction bipolar transistors. (Presentation E4.2, T.R. Fullowan et al., ATET Bell

Laboratories.)
Akasaki et al.) succeeded in making conducting p-type GaN and demonstrated bright blue injection luminescence at room temperature.

\section{Ferroelectric Thin Films are \\ Now Being Integrated with \\ Semiconductor Technology}

(See MRS Symposium Proceedings Vol. 243)

Reports of dramatic progress in ferroelectric thin film device integration and vapor phase deposition techniques highlighted Symposium I, Ferroelectric Thin Films II. Sophisticated integration of ferroelectric thin films with semiconductor technology was demonstrated for pyroelectric detectors, pressure sensors, and ULSI DRAMs. Highly oriented, in some cases epitaxial, ferroelectric thin films were fabricated by a variety of vapor deposition techniques. Ferroelectric properties of vapor deposited PZT thin films exceeded those of the best bulk polycrystalline materials.

Recent developments of integrated ferroelectric-semiconductor devices were typified by the meshing of sol-gel deposited PZT films with CMOS silicon technology to form integrated pyroelectric devices, as reported by Dennis Polla and co-workers from the University of Minnesota. Yields of $100 \%$ were claimed for integrated silicon wafers containing 11,000 transistors and 4,000 PZT capacitors of $30 \mu \mathrm{m} \times 30 \mu \mathrm{m}$ dimensions. Pyroelectric operation at ambient with detectivities ( $D^{\prime}$ ) of $10^{9}\left(\mathrm{cmHz}^{1.2}\right) / \mathrm{W}$ and megahertz operation was predicted. Other interesting examples of device integration included a report of a 64 Mbyte DRAM using barium strontium titanate films by Shogo Matsubara of NEC.

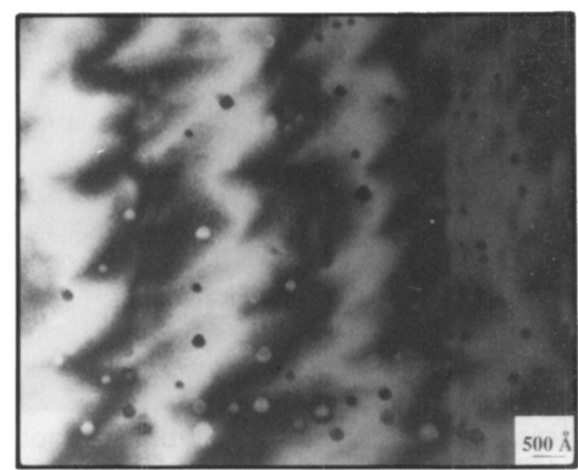

Cross-sectional transmission electron micrograph of GaAs:As. This sample was annealed at $900^{\circ} \mathrm{C}$ to enhance the size of the arsenic precipitates. (Presentation F2.2, J.M. Woodall et al., IBM.) 
Further, Michael Sayer of Queens University reported on optical fibers coated with thin, interdigitated PZT film structures for medical imaging devices. Duane Dimos, Sandia National Laboratories, presented techniques to obtain large optical retardations $\left(>60^{\circ}\right)$ in PZT thin films as thin as $0.5 \mu \mathrm{m}$. These retardations are suitable for optical disk storage applications.

G.J. Dormans of Philips Research Laboratories presented the best PZT thin film properties for films fabricated by organometallic chemical vapor deposition (OMCVD) to date. Remanent polarizations in excess of $60 \mu \mathrm{C} / \mathrm{cm}^{2}$ and $50 \mu \mathrm{C} / \mathrm{cm}^{2}$ were measured for tetragonal PZT 23/77 and rhombohedral PZT 57/33 films, respectively. Switching speeds in the ns regime and functional remanent polarization after $10^{11}$ cycles were demonstrated. These PZT films were deposited at $700^{\circ} \mathrm{C}$ on platinized silicon wafers, indicating compatability with present silicon technology. OMCVD films may prove superior to chemically prepared films for high density device integration and a range of optical applications. Several other significant developments in the vapor phase deposition of highly oriented or epitaxial ferroelectric films were reported. R. Ramesh and coworkers from Bellcore have developed a pulsed laser deposition technique to fabricate highly (100) oriented, PZT 20/80 films on YBCO superconductor electrodes. The rocking curves of the PZT (002) x-ray diffraction peak were $0.6^{\circ} \mathrm{FWHM}$, indicating near-single-crystal quality. These films exhibited good ferroelectric properties and useable polarizations beyond $10^{10}$ electrical cycles. Angus Kingon and co-workers from North Carolina State University reported on the development of epitaxial $\mathrm{KNbO}_{3}$ films at $500^{\circ} \mathrm{C}$ by ion beam deposition techniques. Electrooptic coefficients of these (100) films were comparable to single crystal $\mathrm{KNbO}_{3}$.

In other developments, Stephen Bernacki of Raytheon described polarization dependent conductivity in PZT films due to the formation of Schottky barriers. Ciaran Brennan of Draper Laboratories developed a model based on Schottky barrier formation and Landau-Devonshire theory that offered an explanation of thermal imprint and transient polarization decay phenomena that plague nonvolatile semi-conductor memory device performance.

\section{Optical Waveguide Materials Offer Materials Challenges \\ (See MRS Proceedings Vol. 244)}

A broad range of materials systems employed for both fiber and planar waveguide applications were examined in Symposium J, Optical Waveguide Materi- als. Systems included oxide and non-oxide glasses, polymers, and crystals in both bulk and thin film configurations. A plenary address "Optical Waveguide Material Challenges for Future Communications" by A.M. Glass of AT\&T Bell Laboratories set the tone for the balance of the meeting.

The explosive growth of this technology has generated a demand for a wide array of electrooptic devices such as fiber lasers, modulators, optical amplifiers, isolators, couplers, sensors, switches, and components that require implementation and processing of new materials.

Perhaps the greatest interest at the symposium was generated by papers devoted to optical amplifiers and fiber lasers. D.J. Di Giovannis of AT\&T Bell Laboratories gave an invited paper outlining the scope, recent progress, and material challenges in the area of rare earth doped oxide and nonoxide glasses, particularly $\mathrm{Er}^{3+}$ based systems for operation at $1.55 \mu \mathrm{m}$ and $\mathrm{Nd}^{3+}$ or $\mathrm{Pr}^{3+}$ doped fibers for future devices to be employed near $1.3 \mu \mathrm{m}$. Recent efforts to enhance the efficiency or pumping of fiber optical amplifiers were reported by J.E. Townsend of Southampton University and B. Pedersen et al. of GTE.

Interest continued on the generation of Bragg gratings in germanosilica-based fibers using short wavelength irradiation. G. Meltz of United Technologies Research Center gave an invited paper describing the basic mechanisms and characteristics of these devices and outlined approaches to enhance these effects.

Work on passive or active planar devices was highlighted by talks on polymeric integrated optics by K. Losch of Alcatel, rare earth doped $\mathrm{LiNbO}_{3}$ by $\mathrm{M}$. DeMicheli of the University of Nice and the deposition of oxide glass waveguides and reflectors on silicon substrates by $\mathrm{H}$. Schneider of Siemens Research Laboratories.

\section{Impedance Spectroscopy Reveals} Microstructure, Transport in Cement (See MRS Proceedings Vol. 245)

Novel cementitious systems and methods of characterizing cement-based materials were highlighted in Symposium $\mathrm{K}$, Advanced Cementitious Systems: Mechanisms and Properties. A series of papers from University of Illinois investigators concerned macro-defect-free cement. Work by P.B. Messersmith and S.I. Stupp showed that intercalated complexes between poly(vinyl alcohol) and calcium aluminate hydrates can form in such systems. The importance of the interphase region (which may contain such complexes) in the fracture behavior was reported by 0.0 . Popoola et al. using TEM observations of specimens strained in-situ.
An overview paper by D.M. Roy (Penn State) was on alkali activated cementitious systems, an area that has attracted much attention recently. H.S. Piertersen and J.M. Bijen (Delft), C.Q. Hua et al. (Sherbrooke) and F.P. Glasser (Aberdeen) discussed ongoing work in specific systems.

A promising method for investigating the development of hydrating cementitious systems is impedance spectroscopy. B. Christensen and co-workers in the Center for Advanced Cement-Based Materials described work in this area in which electrical properties are combined with pore solution composition to provide information about pore structure and pore conductivity. The experimental results are compared with the predicted behavior determined from computer models. The agreement is good. Thus, impedance spectroscopy may give information not only about the developing microstructure, but also about the transport properties of the material.

\section{Nondestructive Analysis Techniques Ease Characterization of Materials for Infrastructure}

In Symposium L, Innovations in the Development and Characterization of Materials for Infrastructure, scientists and engineers of various disciplines presented work on structural and nonstructural uses of cements, metals, polymers, and with bituminous and concrete pavements. A number of new nondestructive techniques for characterizing cements, concretes, and asphalt pavements, and new materials for the infrastructure were highlighted.

R.J. Lee of R.J. Lee Group, Inc. described the use of advanced electron optical techniques and gamma-ray tomography to carry out in-situ analysis of damage in concrete structures. An overview of commercially available equipment to carry out this analysis was presented.

Costas Synolakis (UCLA) discussed advances made at the University of Southern California in developing asphalt core tomography (ACT). ACT has advanced to routine use in forensic studies of the detailed composition of asphalt cores, to determine whether certain contract specifications have been met, or to investigate the cause of failure of asphalt pavements. Synolakis presented a computer animated display of the experiments to demonstrate how the technique is used to study and relate the interior images of an asphalt core before and after loading, and to simulate crack formation during cyclic loading.

Robert Pearson (Tri-Valley Research) talked about nuclear magnetic resonance (NMR) spectrometers as a process control 
device in an asphalt hot mix plant. NMR's potential application to the study of the rheology of both neat asphalt, and asphalt mixed with aggregate, gives the technique much promise as a process control device in the highway industry.

New cementitious materials that are potentially useful in the infrastructure were discussed in a series of papers. New materials included a new sulfate-rich cement, a pore reduced cement (cement in which the pores and excess water are removed through the use of high pressure), the production of high strength cement-based materials through the use of silica fume and ground blast furnace slag, and the use of metakaolin clay to reduce efflorescence in a cementitious formulation.

\section{Ni-Al Gains Attention as Shape Memory Alloy (See MRS Proceedings Vol. 246)}

Materials scientists, engineers, physicists, and even mathematicians gathered for Symposium M, Shape-Memory Materials and Phenomena, to cover fundamentals and applications of a wide variety of shape memory materials and the martensitic transformations on which they are based.

One noticeable trend in the present symposium was that there were many papers on $\mathrm{Ni}-\mathrm{Al}$ alloys, in addition to those on more traditional $\mathrm{Ti}-\mathrm{Ni}$ alloys. Of the 77 papers in the symposium, 16 were on $\mathrm{Ni}-\mathrm{Al}$ alloys. The reason is twofold. First, recent neutron scattering experiments revealed a close relationship between the phonondispersion relationship in the parent phase and the structure of the martensite. This relationship gives insight into the microscopic mechanism of the transformation. Second, the alloy has potential as a high temperature shape memory alloy (SMA). Although Ti-Ni alloys are very useful as SMAs, they can be used only up to $100^{\circ} \mathrm{C}$. $\mathrm{Ni}$-Al alloys have high transformation temperatures and high oxidation resistance, so they may function at higher temperatures. The symposium showed that first principle calculations can now give detailed information on energies and atomic forces essential in the analysis of the microscopic transformation mechanism. One computer simulation using molecular dynamics made the extremely rapid transformation visible, which was otherwise impossible by experiment. The result showed the heterogeneous nature of nucleation at special sites and the large amplitude localized soft mode, which may be the origin for the "tweed pattern" characteristic of this alloy. Various compositions of $\mathrm{Ni}-\mathrm{Al}-\mathrm{X}(\mathrm{X}=\mathrm{Fe}, \mathrm{B}, \mathrm{Mn}$, etc.) alloys were made to improve ductility, which, at room temperature, is nil. The result seems to indicate that Ni-Al-Mn alloys are useful as high temperature SMA.

Another new emphasis in the symposium was on thin film SMAs prepared by sputtering and rapid quenching, which are important for micro-actuators and micromachines. Although the results were preliminary, several problems such as the effect of stress from substrates and that of oxygen, etc., were pointed out.

There were many other interesting papers as single contributions, such as the use of SMA for adaptive structures, the structure determination of $\xi_{2}$ martensite in a Au-Cd alloy, and the application of SMA for a rock breaker.

\section{Fullerenes Continue to Show Off Their New Chemistry \\ (See MRS Proceedings Vol. 247)}

Two special evening sessions on the chemical modifications and applications of buckminsterfullerenes were held as part of Symposium N (Electrical, Optical, and Magnetic Properties of Organic Solid State Materials). There were 17 oral and 37 poster papers presented on fullerenes. The evening oral session on December 4 attracted a crowd of 550 with about 50 remaining until the session ended, shortly after midnight. The excitement over fullerenes seems to grow continuously even a year after the announcement of the synthesis and isolation of a macroscopic quantity of buckyballs last September. Many new research groups are now committed to this activity.

Reports about the superconductivity of fullerene-based materials included different aspects of chemistry and physics of metal doped $C_{60}$. The superconducting $T_{C}$ still remains at roughly $30-45 \mathrm{~K}$. However, the stability of the alkali doped $\mathrm{M}_{3} \mathrm{C}_{60}$ is a major concern. Its high reactivity towards oxygen or moisture upon exposure to air may hinder some potential applications.

New functionalization chemistry was reported to modify fullerenes into a novel class of materials for potential technological uses. Both nucleophilic substitution and photochemically generated radical addition were described for adding multiple diphenyl and benzyl substitutions on fullerene molecules. In addition to these two synthetic routes, it was demonstrated that the electrophilic substitution on fullerenes, in either aqueous acid chemistry or nonaqueous solution through epoxidation reaction or nitronium chemistry, can indeed provide a versatile synthetic methodology to introduce multiple polar substituents such as hydroxy groups directly on ful- lerenes. Other chemistry includes the generation of radical cations of fullerenes in strong acids followed by nucleophilic trapping to incorporate alkoxy groups. The synthesis of a fullerene based polymer or oligomer seems to be possible using paraxylene diradicals as molecular linking agents.

In the separation of larger fullerenes, by using $\mathrm{CS}_{2}$ and size exclusion chromatography, a fullerene size up to 160 carbons can be isolated. Tubular fullerenes, proposed to be axially concentric closed graphitic tubes drew substantial interest. Theory predicts that such tubules, depending on the tube diameter, length, and organization of the hexagons, exhibit conducting, semiconducting, or insulating properties, and thus may be used as building blocks for novel electronic devices. However, the difficulty of synthesizing and separating specific sizes and shapes of tubules is still a major obstacle.

There were reports on characterizations of endohedral complexes of lanthanum with fullerenes as La@ $\mathrm{C}_{82}$. The possible formation of fullerenes containing up to

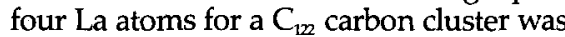
also described. Evidently, the chemical structure of $\mathrm{C}_{60} \mathrm{O}$ is now proved to be fullerene expoxide instead of fullerene oxide. The use of $C_{0}$ as a precursor for diamond nucleation in a plasma CVD experiment represents another aspect of fullerene application. It was reported that the nucleation enhancement is about 10 orders of magnitude over that on an untreated silicon surface, comparable to that for a diamond grit polished surface.

In the panel discussion session in the evening of December 5, discussions and concern over the issue of safe handling of fullerene carbon clusters had been addressed. There were controversial comments on the level of toxicity of nonsubstituted fullerenes.

As to the viewing of the technological value of fullerenes, expectations are growing for finding specific chemical applications of functionalized derivatives since more functionalization chemistry is now available. Meanwhile, to minimize the cost of materials, it was suggested that catalysis may be one promising route to pursue.

\section{Complex Fluids Go Non-Equilibrium \\ (See MRS Proceedings Vol. 248)}

One of the exciting areas now being studied in the field of Complex Fluids (Symposium O) is how the fluids behave under non-equilibrium conditions and more specifically, under uniform steadystate shear flow. While previous work concentrated on equilibrium properties, the 
same experimental techniques, with important new technical innovations, are now being applied to studying the properties under shear flow. P.L. Dixon et al. (Exxon) presented their work on semi-dilute polymer solutions using elastic light scattering to probe the anisotropy of concentration fluctuations on long length scales. C.R. Safinya et al. (Exxon) presented their work on thermotropic and lyotropic liquid crystalline systems under steady state shear using $x$-ray scattering techniques, probing the effect of shear and studying the anisotropy of the structure on shorter length scales. P. Tong et al. (Oklahoma State University) used photon correlation spectroscopy to probe turbulent drag reduction in a dilute polymer solution. G. Fuller (Stanford University) discussed the development of optical rheometry techniques as applied to polymer melts and blends. Theoretical techniques are also being used now to study complex fluids under shear, both to explain recent experiments and to suggest new ones. S. Milner (Exxon) presented work on polymer solutions and R. Bruinsma et al. (UCLA) presented their work on fluctuations in layered systems.

\section{Methods to Synthesize and Process Ceramics are Refined and Expanded (See MRS Proceedings Vol. 249)}

The symposium on Synthesis and Processing of Ceramics $(Q)$ began with presentations concerning the synthesis of preceramic polymers and their applications. Most of the research on preceramic polymers has been devoted to the synthesis of non-oxide ceramics, particularly $\mathrm{SiC}$, $\mathrm{Si}_{3} \mathrm{~N}_{4}$, and composite materials. Several presentations discussed the synthesis of oxide ceramics from preceramic polymers which is an alternative to the sol-gel approach. Precipitation and hydrothermal methods for synthesizing powders from solutions were also presented, and methods to accelerate the crystallization of particles in aqueous solutions were presented in an invited presentation. A number of papers were presented concerning the synthesis of powders by combustion synthesis and gas phase methods, including thermal decomposition of aerosols and the decomposition of volatile precursors by microwave plasmas. The powders produced from microwave plasmas were reported to be very reactive and to sinter at very low temperatures. Coatings and homo- and heteroepitaxial films can be found from metalorganic precursors.

Processing sessions covered all aspects of solution-based methods for processing ceramic powders. Processing presentations described interparticle forces that control the stability of dispersions, polymers used in steric stabilization, and methods to control their attachment to and orientation on particle surfaces. Shortrange repulsive forces, measured experimentally but not explained by the theories, are being exploited for processing ceramic powders, and processing approaches can take advantage of these forces. Contributed papers were presented on controlling the particle surface chemistry to obtain good dispersions of ceramic powders.

One session was devoted to methods for forming and characterizing textured microstructures including processing approaches for controlling the microstructure of superconducting ceramics and ceramicceramic composites. The removal of solvents and processing aids are also important processing steps, and presentations discussed advances in understanding the drying of granular ceramic bodies. Other papers discussed processing with preceramic polymers, and removing polymers from injection molded parts.

Sintering and the evolution of microstructure during densification of ceramic materials were presented, along with results on the effects of additives. In another session, the problems of densifying ceramic-ceramic composites were discussed. An invited presentation gave a review of progress on using transient viscous phases to densify ceramic composite materials. Reaction bonding and combustion synthesis methods were also discussed, along with progress in improving the properties and understanding the scientific issues involved in reaction-bonded silicon nitride.

In an interesting and well-attended session, some of the newer approaches to the processing and synthesis of ceramic materials were presented. Invited papers discussed biomimetic materials, processing colloidal size particles, self-assembled layered ceramic nanocomposites, and the synthesis of organic/inorganic semiinterpenetrating networks (ceramers).

\section{CVD Fine Tuned to Produce Better \\ Refractory Metals and Ceramics \\ (See MRS Proceedings Vol. 250)}

The symposium on the Chemical Vapor Deposition of Metals and Ceramics (Symposium $R$ ) was very well attended with approximately 70 papers presented over a three-day period. The meeting led off with a session on the fundamentals of CVD, highlighted by Claude Bernard of the Laboratoire des Materiaux et du Genie Physique in France and Suleyman Gokoglu of the NASA Lewis Research Center. Bernard discussed the thermochemistry of CVD on the choice of halide gas species, noting the importance of thermochemical analysis in the choice of reactants. Gokoglu, talking on CVD modeling for high temperature materials, commented on the relative difficulty of modeling and the hierarchy of what is understood today. Other papers on fundamentals discussed kinetics and mass transport in CVD, with an emphasis on silicon carbide deposition.

A very interesting session on in-situ diagnostics in CVD presented information on techniques such as spectroscopic ellipsometry and laser light scattering. The reports emphasized both the importance of in-situ diagnostics and how this area is just now emerging.

The session on microstructure-processproperty relationships revealed the ability to control the CVD coatings to a level not seen before. Coatings of more than one phase are now routinely deposited to ob-
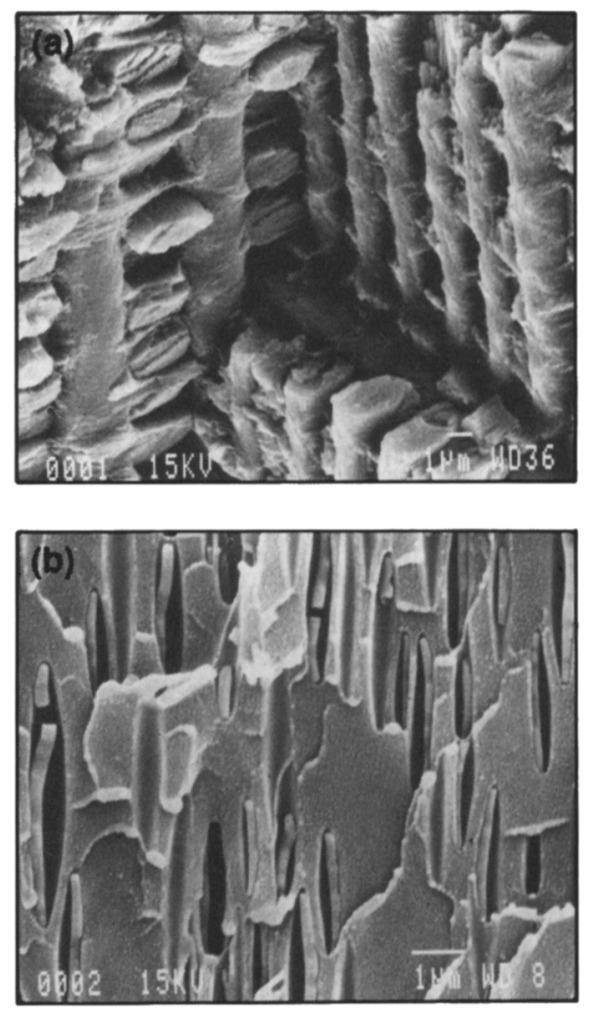

Structure of a rat's tooth with rods of mineral in cross-ply layers and an effort to form similar rods of titania in a plastic matrix. (a) Section of a rat's tooth showing the crossed layers of rods of hydroxyapatite giving a structure which is hard like a ceramic, but much tougher. (b) Section of a composite. A partial mimic of a rat's tooth. Long particles of titanium dioxide are grown within a confining and directing polymer matrix. (Presentation Q8.1, Paul Calvert, Arizona Materials Laboratories.) 
tain material with a variety of properties. Chemical vapor infiltration to produce composite materials was the subject of another session, and it included considerable discussion of the modeling of the infiltration process, which is becoming quite advanced.

The session on organometallic CVD focused on a number of precursor issues and the mechanisms of deposition. In light of the interest in high $\mathrm{T}_{C}$ superconductors, a good bit of the discussion revolved around copper precursors.

The final session was on the CVD of diamond, and Karl Spear of Pennsylvania State University pointed out that more attention needs to be paid to surface reactions to understand the deposition process. Other papers reported on nucleation studies, the nature of the plasma in plasma CVD of diamond, and novel techniques for growth.

\section{Levitation and Hot Isostatic Forging Spur Gas Pressure Processing}

(See MRS Proceedings Vol. 251)

Recently, the market for hot isostatic pressing (HIP) equipment has been stagnating, however, the papers presented in Symposium S, Gas Pressure Effects on Materials Processing and Design, showed that high pressure research using $\mathrm{HIP}$ is still very much alive, with new research opportunities arising.

Several papers showed that HIP is still an important process for one of its first applications: densification. It was reported that high temperature application materials could be fully densified using HIP. Densification of glasses without crystallization was shown to produce interesting optical properties. A continuing trend is the search to reduce the cost of the HIP process, either by using hybrid systems, like rate controlled sintering (RCS) with $\mathrm{HIP}$, or by new processes. A new economic densification process is hot isostatic forging (HIF), which has floor-to-floor cycle times of only minutes compared to hours for HIP. Pressures up to 60,000 psi (414 MPa) are possible, and only moderate temperatures $\left(800^{\circ} \mathrm{C}\right)$ are necessary to reach full density for materials which normally require about $1200^{\circ} \mathrm{C}$ to reach full density by HIP.

Apart from new materials which can only be densified or upgraded by HIP, a total new field of applications was presented in this symposium. Probably the most important one is the levitation of material using high pressure gas. Due to the high density of gasses at high pressures (e.g., the density of Ar-gas at $100 \mathrm{MPa}$ is the same as for water), material can be lifted and begun to float. This levitation can be used to make prefect spheres or to sinter or melt without contamination from the crucible material. This process may offer a cost-effective alternative to expensive microgravity experiments.

Another important new field is containerless HIP to make stronger porous materials. These materials are believed important for future filter applications.

Controlling partial and total pressures during sintering leads to other interesting applications. By introducing small amounts (ppm) of oxygen in the inert Asgas used during HIP, it was possible to produce $\mathrm{Mn}-\mathrm{Zn}$ ferrites without powder embedding, normally used to control partial pressures. The possibility of high total and oxygen partial pressures makes it also possible to obtain, for example, Y-Ba-Cu-O, 124 superconducting phase.

\section{New Strategies Emerge for Synthesis and} Characterization of Catalysts

The symposium on New Strategies for the Synthesis and Characterization of Catalysts (Symposium U) encompassed a broad range of topics with sessions devoted to metal cluster chemistry and highly dispersed structures, zeolites and microporous materials, oxide catalysis, transient characterization methods, and electron microscopy. Six presentations by invited speakers were complemented by 61 contributed presentations plus posters.

Highlights from the sessions related to metals included new developments in oxidation catalysis and sonochemistry, the preparation of well-defined clusters in zeolites, the generation of fine particles from metal hydrides and carbonyls via electron and laser stimulated decomposition, and the building of multilayer, multicomponent model catalysts with well-defined structures on single crystal substrates.

New insight into the structure and catalytic properties of acid sites in zeolites and silicon-aluminum phosphates suggested applications for these materials in acid catalyzed hydrocarbon conversions. Specific preparation conditions were emphasized in the sessions on oxide catalysis in order to control bulk and surface structures for stabilizing dispersed phases and for tailoring selectivity in a variety of oxidation, carbonyl methathesis, polymerization, denitrogenation, dehydrogenation, and $\mathrm{SO}_{x}-\mathrm{NO}_{x}$ reduction reactions. Presentations detailing the use of isotopic transients and double rotation NMR in catalyst characterization received considerable interest as did studies of adsorption phenomena on semiconducting catalyst particles using the Hall effect.
Several exciting developments in electron microscopic characterization of catalysts included the use of imaging SIMS for quantitative analysis, the definition of oxide surface structure from modest resolution SEM images, the tendency for quasimelting in small metal particles prior to coalescence, and the use of spherical alumina supports to obtain high resolution images of dispersed oxides and sulfides.

It is now anticipated that proceedings of this symposium will be published as a special issue of Catalysis Letters in mid-1992.

\section{Multiple Scattering Theory Helps Deter- mine Electronic and Surface Structure (See MRS Proceedings Vol. 253)}

Symposium V, Applications of Multiple Scattering Theory to Materials Science, included 63 papers divided among 8 sessions, including a joint session with symposium P (Disordered Systems). The field appears to be in a stage of rapid advancement especially in the areas of full potential multiple scattering theory (MST) and the extension of MST to include relativistic effects which are important for understanding magnetic anisotropies in materials. Especially impressive work described the use of multiple scattering theory and low energy electron diffraction to solve for surface structures as well as the use of multiple scattering theory and photoemission to determine the electronic structure of a wide range of materials.

\section{Workshop Focuses on Specimen \\ Preparation for Transmission Electron Microscopy \\ (See MRS Proceedings Vol. 254)}

Virtually all symposia at MRS meetings are filled with transmission electron microscopic analyses. TEM specimen preparation is often the factor that influences the success or failure of a particular scientific investigation. Since the art of TEM specimen preparation is evolving constantly, it was deemed important that workshops on TEM specimen preparation be presented periodically.

The present workshop, third in a series, was a half-day platform session featuring invited speakers addressing various topics in TEM specimen preparation. Speaker invitations were made to those researchers making important contributions to specimen preparation in commercial specimen preparation theory, instrumentation, and applications in the physical sciences. In the theoretical category, A. Barna of the Hungarian Institute of Physics reported on the theory of ion milling and the new ion milling instrumentation evolved from his work. In the instrumentation category, R. 
Alani, from Gatan reported on their precision ion polisher and their rastered ion beam thinning unit. Advancements in dimpling were covered by P. Fischione, of E.A. Fischione, Inc., and V. Carlino of VCR Group. Fischione also discussed a new ultrasonic cutter. Preparing TEM specimens using a Ga source focused ion beam tool was the subject of D. Basile's (Intel) talk in the applications area.

A well-attended evening poster session attracted 22 contributions showing new and evolving methods for preparing various classes of specimens. The largest number of poster contributions were on various aspects of preparing semiconductor specimens for TEM analysis. The paper by J. Benedict et al. (IBM) reported routine preparation of pre-defined, micron-sized, cross section samples, while J. McCaffrey (National Research Council of Canada) showed how to prepare specimens using a small-angle cleaving method. Another group of posters presented methods for preparing particulate and fibrous samples. Other posters focused on new or improved tools for specimen preparation, including a dedicated focused ion beam tool for TEM specimen preparation, as displayed by $\mathrm{R}$. Clampitt (Oxford Applied Research).

\section{Hierarchically Structured Materials Show Lessons from Nature \\ (See MRS Proceedings Vol. 255)}

When materials are manufactured with an emphasis on tailoring properties through microstructural control, the extent of this microstructural control is generally at a specific length scale. In contrast, many naturally occurring materials are very complex in structural design at spectrum of length scales varying from atomic to macroscopic dimensions and with unique hierarchical architectures.

The hierarchical architecture of cellulose aggregates in wood or collagen-based aggregates in biocomposites (e.g., skin, cartilage, bone, and seashell) are excellent examples of nature's way of designing composites for multifunctional applications by efficient and ecologically balanced process methodologies. These hierarchically structured materials display unique properties affected by processes at all levels of this hierarchical spectrum. The intent of Symposium Z, Hierarchically Structured Materials, was to focus on the unique properties of naturally occurring hierarchically structured systems and to critically evaluate techniques and processing procedures for assembling synthetic hierarchically structured materials to achieve unique properties that cannot be achieved by other methods.

The symposium consisted primarily of invited presentations held in eight sessions. The session on structures, properties, and design rules in natural

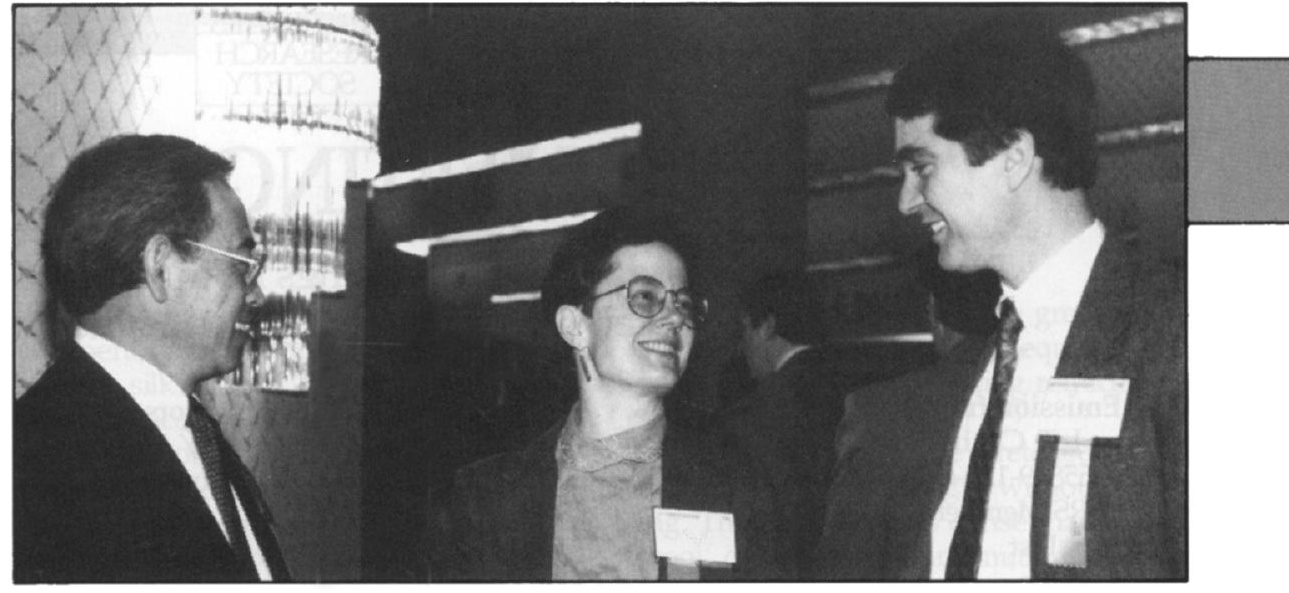

Chairs of the 1991 MRS Fall Meeting (left to right): Man H. Yoo, Julia M. Phillips, and Michael M.J. Treacy.

hierarchical materials, examined structures in tendon, seashells, and silks, and discussed the basis of hierarchy in these biological hard and soft tissues. Structures and design rules in synthetic hierarchical materials concentrated on polymer blends, liquid crystals, and steels, and the possible pathways to produce these equilibrium and metastable equilibrium hierarchical structures. Composite structures and mechanical properties focused on the mechanical property-microarchitectural relationships in both synthetic and natural composites, with a special emphasis on lamination. How structural evolution may be controlled from nanometer to higher scales of dimensions was covered in the session on pattern formation in synthetic and natural composites. Electronic, optical, and magnetic properties of hierarchically structured materials addressed how these nonlinear properties can be controlled by the hierarchy in the structure. Scaling and structural characterization of hierarchical natural and synthetic structures included statistical mechanics of phase transformations in hierarchical structures, mathematical descriptions of hierarchical tilings, scaling relationships in cellular structures, and imaging (atomic to millimeter scale) and spectroscopical characterization of hierarchical natural and synthetic materials. Processing of synthetic-hierarchical structures included vesicle-mediated ultrafine particle formation, self-assembled layers, and a biomimetic approach to synthetic composite design and processing (i.e., biomimicking). The last session on molecular design of hierarchical structures included natural examples and the initial work on the genetic design of proteins and self-assembled macromolecules and their structural buildup, a first step towards bioduplication. Symposium $Z$ was dedicated to the memory of Donald R. Ulrich.

\section{Workshop Explores Ways to Encourage Young Scientists}

An educational workshop was held which was co-sponsored by the University Materials Council. During the workshop, various approaches to materials education, from precollege education to advanced graduate level, were exchanged by educators and scientists across the country. Included among the presentations was the MRS Grassroots Education effort in getting MRS members involved in the enhancement of precollege science education. Other presentations dealt with the integration of materials chemistry, synthesis and processing, and design into undergraduate programs. For the graduate level, the presentations focused on the interdisciplinary aspects of materials education, and core curriculum requirements. In addition, a presentation was made on $\mathrm{Na}$ tional Science Foundation plans to expand its support for materials education at the undergraduate and graduate levels. For more information, contact Professor Reza Abbaschian, Department of Materials Science and Engineering, University of Florida, phone (904) 392-6609

MIRIS

\section{THANKS...}

To all the symposium organizers who contributed summaries for the 1991 MRS Fall Meeting report published in this issue.

The MRS Bulletin and the organizers welcome your comments and suggestions for future MRS meeting coverage. Contact: E.L. Fleischer, Technical Editor, MRS Bulletin, Materials Research Society, $9800 \mathrm{McKnight}$ Road, Pittsburgh, PA 15237 USA; phone (412) 367-3036; FAX (412) 367-4373. 


\section{FALL MEETING PROCEEDINGS}

\section{Place your order today for proceedings from the 1991 Fall Meeting in Boston. You'll receive your books as soon as they are published.}

Light Emission from Silicon (Symp. AA)

S.S. Iyer, L.T. Canham, R.T. Collins

ISBN: 1-55899-150-6 Volume 256 B

$\$ 45$ MRS Members

$\$ 52$ U.S. List

$\$ 67$ Foreign

Phase Formation and Modification by

Beam-Solid Interactions (Symp. A)

G.S. Was, L.E. Rehn, D. Follstaedt

ISBN: 1-55899-129-8 Volume 235 B

\$44 MRS Members

$\$ 50$ U.S. List

$\$ 65$ Foreign

Photons and Low Energy Particles in

Surface Processing (Symp. B)

C. Ashby, J.H. Brannon, S. Pang

ISBN: 1-55899-130-1 Volume 236 B

$\$ 40$ MRS Members

$\$ 49$ U.S. List

$\$ 64$ Foreign

Interface Dynamics and Growth

(Symp. Ca)

K.S. Liang, M.P. Anderson, R.F. Bruinsma,

G. Scoles

ISBN: 1-55899-131-X Volume 237 B

$\$ 44$ MRS Members

$\$ 50$ U.S. List

$\$ 65$ Foreign

Structure \& Properties of Interfaces in

Materials (Symp. Cb)

W.A.T. Clark, C. L. Briant, U. Dahmen

ISBN: 1-55899-132-8 Volume 238 B

$\$ 47$ MRS Members

$\$ 54$ U.S. List

$\$ 70$ Foreign

Thin Films: Stresses and Mechanical

Properties III (Symp. D)

W.D. Nix, J.C. Bravman, E. Arzt,

L.B. Freund

ISBN: 1-55899-133-6 Volume 239 B

$\$ 47$ MRS Members

$\$ 53$ U.S. List

$\$ 68$ Foreign

Advanced III-V Compound Semiconductor Growth, Processing and Devices (Symp. E)

S.J. Pearton, D.K. Sadana, J.M. Zavada

ISBN: 1-55899-134-4 Volume 240 B

$\$ 47$ MRS Members

$\$ 53$ U.S. List

$\$ 68$ Foreign

Low Temperature (LT) GaAs and Related Materials (Symp. F)

G.L. Witt, R. Calawa, U. Mishra, E. Weber

ISBN: 1-55899-135-2 Volume 241 B

$\$ 44$ MRS Members

$\$ 50$ U.S. List

$\$ 65$ Foreign
Wide Band-Gap Semiconductors (Symp. G) T.D. Moustakas, J.I. Pankove, Y. Hamakawa ISBN: 1-55899-136-0 Volume 242 B

$\$ 44$ MRS Members

$\$ 50$ U.S. List

$\$ 65$ Foreign

Ferroelectric Thin Films II (Symp. I)

A.I. Kingon, E. R. Myers, B. Tuttle

ISBN: 1-55899-137-9 Volume 243 B

$\$ 40$ MRS Members

$\$ 49$ U.S. List

$\$ 64$ Foreign

Optical Waveguide Materials (Symp. J)

M.M. Broer, H. Kawazoe, G.H. Sigel,

R. Th. Kersten

ISBN: 1-55899-138-7 Volume $244 \mathrm{~B}$

$\$ 47$ MRS Members

$\$ 53$ U.S. List

$\$ 68$ Foreign

Advanced Cementitious Systems:

Mechanisms and Properties

(Symp. K)

F.P. Glasser, P.L. Pratt, T.O. Mason,

J.F. Young, G.J. McCarthy

ISBN: 1-55899-139-5 Volume $245 \mathrm{~B}$

$\$ 44$ MRS Members

$\$ 50$ U.S. List

$\$ 65$ Foreign

Shape-Memory Materials and Phenomena - Fundamental Aspects and Applications (Symp. M)

C.T. Liu, M. Wuttig, K. Otsuka,

H. Kunsmann

ISBN: 1-55899-140-9 Volume 246 B

$\$ 38$ MRS Members

$\$ 44$ U.S. List

$\$ 57$ Foreign

Electrical, Optical, and Magnetic Properties of Organic Solid State Materials (Symp. N) L. Y. Chiang, A.F. Garito, D.J. Sandman ISBN: 1-55899-141-7 Volume 247 B

$\$ 44$ MRS Members

$\$ 50$ U.S. List

$\$ 65$ Foreign

Complex Fluids (Symp. O)

D. Weitz, E. Sirota, T. Witten, J. Israelachvili ISBN: 1-55899-142-5 Volume 248 B

$\$ 40$ MRS Members

$\$ 49$ U.S. List

$\$ 64$ Foreign
Synthesis and Processing of Ceramics: Scientific Issues (Symp. Q)

W.E. Rhine, T.M. Shaw, R.J. Gottschall,

Y.Chen

ISBN: 1-55899-143-3 Volume 249 B

\$42 MRS Members

$\$ 49$ U.S. List

$\$ 64$ Foreign

Chemical Vapor Deposition of Refractory

Metals and Ceramics II (Symp. R)

T.M. Besmann, B.M. Gallois, J. Warren

ISBN: 1-55899-144-1 Volume 250 B

\$38 MRS Members

$\$ 44$ U.S. List

$\$ 57$ Foreign

Pressure Effects on Materials Processing and Design (Symp. S)

K. Ishizaki, E. Hodge

ISBN: 1-55899-145-X

$\$ 38$ MRS Members

$\$ 44$ U.S. List

$\$ 57$ Foreign

Tissue-Inducing Biomaterials (Symp. T) M. Flanagan, L. Cima, E. Ron

ISBN: 1-55899-146-8 Volume 252 B

$\$ 45$ MRS Members

$\$ 53$ U.S. List

$\$ 68$ Foreign

Application of Multiple Scattering

Theory to Materials Science (Symp. V)

W.H. Butler, P.H. Dederichs, A. Gonis,

R. Weaver

ISBN: 1-55899-147-6 Volume 253 B

$\$ 45$ MRS Members

$\$ 53$ U.S. List

$\$ 68$ Foreign

Specimen Preparation for Transmission Electron Microscopy of Materials III (Symp. W)

R. Anderson, J. Bravman, B. Tracy

ISBN: 1-55899-148-4 Volume 254 B

$\$ 42$ MRS Members

$\$ 49$ U.S. List

$\$ 64$ Foreign

Hierarchically Structured Materials (Symp. Z)

I.A. Aksay, E. Baer, M. Sarikaya, D.A. Tirrell ISBN: 1-55899-149-2 Volume 255 B

$\$ 42$ MRS Members

$\$ 49$ U.S. List

$\$ 64$ Foreign 